\title{
Metastatic Malignant Thymoma to the Abdomen: A SEER Database Review and Assessment of Treatment Strategies
}

\author{
J. Matthew Helmª, Dan Lavy ${ }^{\mathrm{b}}$, Jazmine Figueroa-Bodine ${ }^{\mathrm{c}}$, \\ Saju Joseph ${ }^{\mathrm{d}, \mathrm{e}, \mathrm{f}}$
}

\begin{abstract}
Background: Thymoma is a neoplasm occurring in 0.15 of 100,000 persons/year. Abdominal metastases are rare. We report the incidence of malignant thymoma (MT) and suggest imaging and treatment options for cases of abdominal metastasis.

Methods: A National Cancer Institute's Surveillance, Epidemiology and End Results database review was conducted to identify MT cases, followed by a literature review examining cases of metastases to the abdomen. Incidence rates were calculated, and symptoms, treatments, size and location of tumors, disease-free interval (DFI), and survival time were recorded.
\end{abstract}

Results: From 1973 to 2008 , a total of 1,588 MT cases were identified (45.4 cases/year), which were extrapolated to 2,724 over 60 years. Incidence has risen from 17 cases in 1973 to 90 cases in 2008, with a larger incidence in males than females $(0.23$ vs. 0.17 per 100,000$)$. There were 25 cases of abdominal metastasis $(0.92 \%), 13$ of which were asymptomatic. There was a wide variety of DFI and survival noted amongst the case reports. Multiple treatment modalities were used.

Conclusions: The incidence of MT is on the rise with a male predominance. All patients should receive routine imaging to look for extrathoracic metastases as half will not have symptoms. All patients with abdominal metastases should be treated using a multimodal approach.

Keywords: Thymoma; Extrathoracic metastasis; Multimodal treatment; Incidence

Manuscript submitted August 15, 2017, accepted September 7, 2017

aTexas Tech University Health Sciences Center School of Medicine, Lubbock, TX, USA

${ }^{\mathrm{b}}$ Cleveland Clinic, Weston, FL, USA

'Yale University School of Medicine, New Haven, CT, USA

dValley Health Systems, Las Vegas, NV, USA

eRoseman University School of Medicine, Henderson, NV, USA

fCorresponding Author: Saju Joseph, Valley Health Systems, 10105 Banburry

Cross Dr Suite 230, Las Vegas, NV 89144, USA.

Email: Saju.Joseph@uhsinc.com

doi: https://doi.org/10.14740/wjon1057w

\section{Introduction}

Thymoma is a neoplasm that consists of thymic epithelial cells that are normally responsible for $\mathrm{T}$ lymphocyte maturation [1]. While primary tumors of the thymus are rare, thymomas account for about $40 \%$ of all mediastinal tumors [2]. Malignant thymomas (MTs) most often spread by local extension and have an overall incidence of only 0.15 per 100,000 persons/year [3]. Metastases are most commonly restricted to the pleura, pericardium, or diaphragm [3]. Extrathoracic metastases to the abdomen are extremely rare. Till recently, only case reports existed. However, in 2012, Vladisla et al [2] presented 35 pathology cases of extrathoracic thymoma. We sought to review the literature and discuss the different treatment options for this disease. We used a publicly available population-based database and literature review to report the incidence of MT abdominal metastasis in the United States, and to suggest criteria for imaging and treatment in patients with this condition.

\section{Materials and Methods}

We used the National Cancer Institute's Surveillance, Epidemiology and End Results (SEER) Program, a publicly available database known for providing cancer statistics in the United States. The program collects data regarding incidence, prevalence and survival within the US population from specific geographical areas, covering about $26 \%$ of African Americans, $41 \%$ of Hispanics, $43 \%$ of American Indians and Alaskan Natives, $54 \%$ of Asians, and $71 \%$ of Hawaiian/Pacific Islanders. Multiple registries, including the Arizona Indian Cancer Registry, Arizona Indians, Cherokee Indians, and the Connecticut and Georgia Centers for Cancer Statistics, provide the data. Over the past 30 years, many groundbreaking scientific studies have been published using this database. We conducted a retrospective review of this database to identify MT cases in the US from 1973 to 2008. We then did a literature review using PubMed of all English language articles involving MT. All cases with metastatic thymoma were reviewed. All cases of metastasis to the abdomen were included in this review. Incidence rates were calculated, and symptoms, forms of treatment, size and location of tumors, disease-free interval (DFI), and time of survival were recorded whenever available. The 


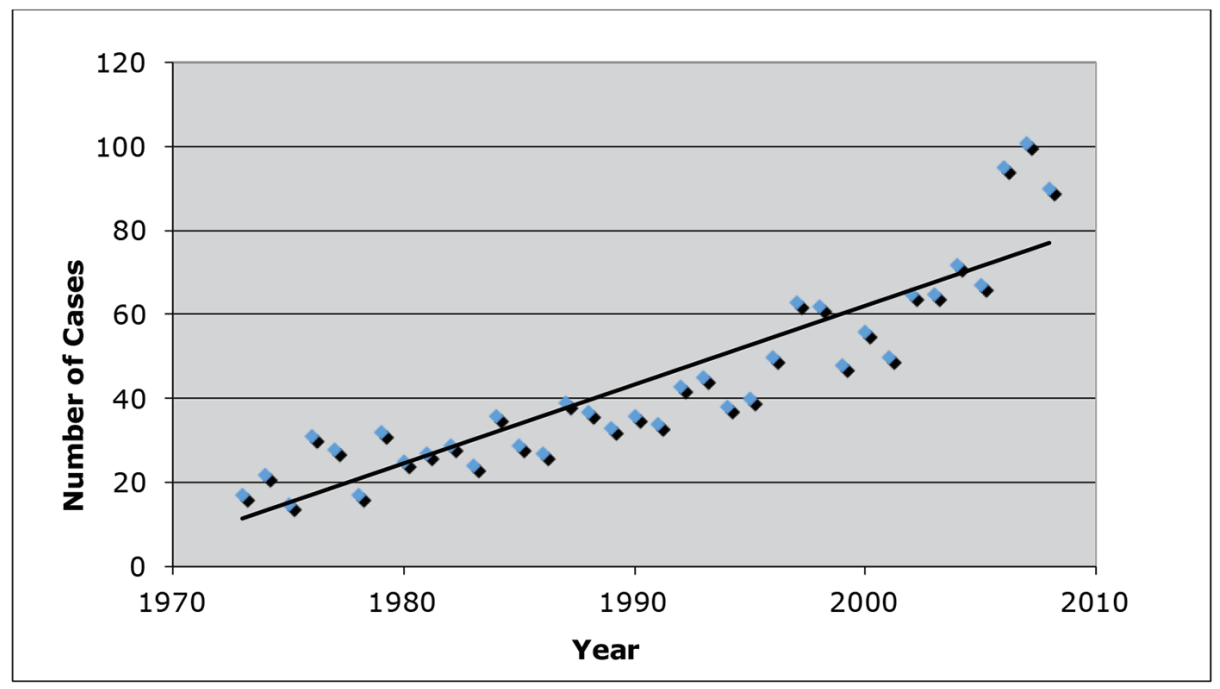

Figure 1. Incidence of malignant thymoma cases from 1973 to 2008 using data from the SEER database.

PubMed literature review spanned a 60-year period.

\section{Results}

The total number of MTs within the United States from 1973 to 2008 was 1,588 cases, an average of 45.4 cases per year. Using this rate, we extrapolated a total of 2,724 cases of MT over a span of 60 years. In conducting a literature review, we identified 25 cases of peritoneal metastasis (an incidence of $0.92 \%$, $25 / 2,724)$, most of which had metastasis to the kidney, liver and gastrointestinal tract.

Since 1973, the overall incidence of $\mathrm{M}$ has risen from 17 reported cases to 90 reported cases in 2008 (Fig. 1). In addition, we found the incidence of MTs to be higher in males than in females ( 0.23 vs. 0.17 per 100,000 persons, 846 vs. $742, \mathrm{P}=$ 0.0002) similar to previous studies (Fig. 2).

Of the 25 peritoneal metastasis cases identified, 13 patients had their disease discovered incidentally and were asymptomatic. Of those with symptoms, abdominal pain was the most frequent symptom noted (12/25). In conducting a review of treatment strategies, 15 of the cases provided treatment information with multiple modalities being used most often. The most common strategy used was a combination of surgery, chemotherapy and radiation treatments $(8 / 15)$. The combinations of surgery and chemotherapy, and chemotherapy and radiotherapy were each used in one of the 15 cases, surgery and radiotherapy alone were both used in two of the 15 cases, and chemotherapy alone was used in one of the 15 cases (Table 1). The largest DFI was 156 months, while the shortest DFI was 3.9 months. The high-

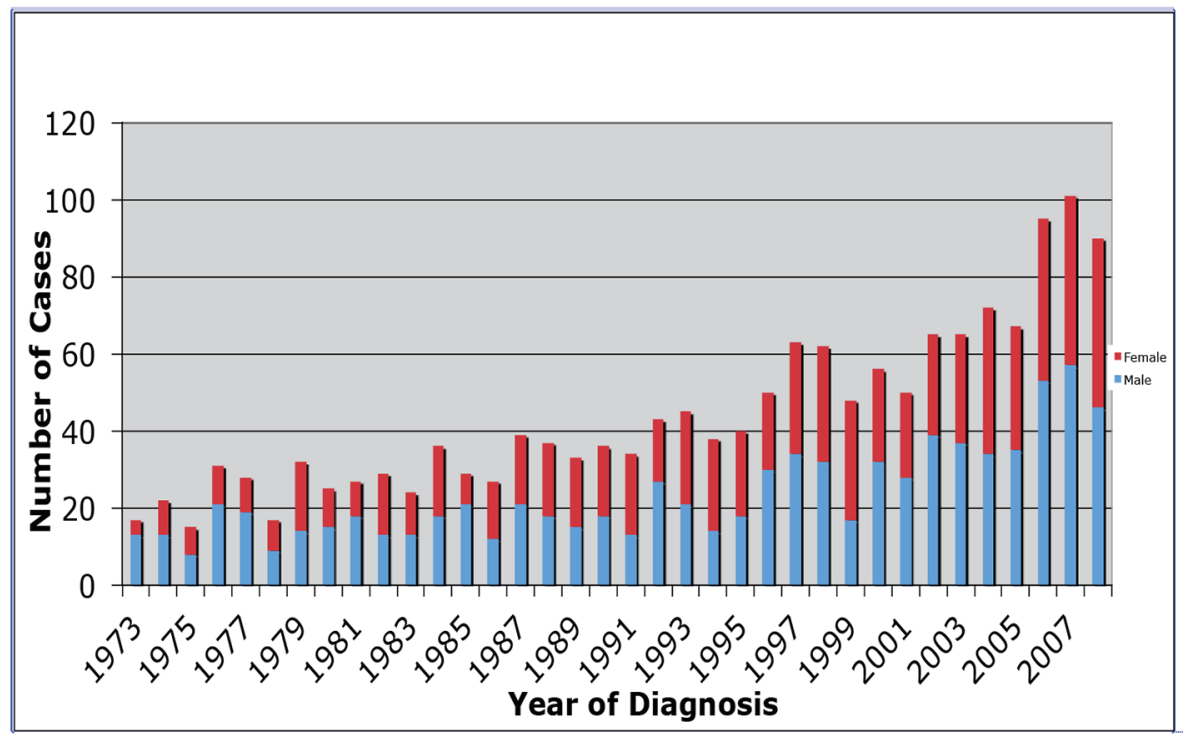

Figure 2. Incidence of malignant thymomas by gender from 1973 to 2008 using data from the SEER database. 
Table 1. Review of the 15 Cases of Extrathoracic Metastasis to the Abdomen Over the Last 60 Years That Provided Treatment Information

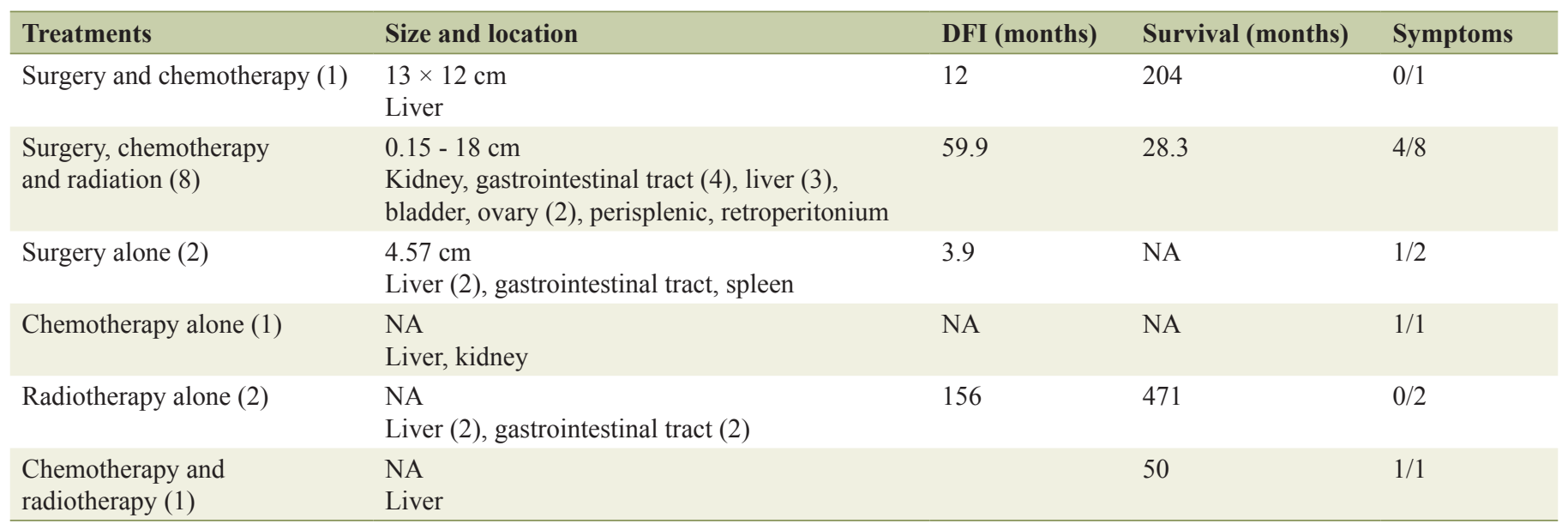

Characteristics of the tumor, treatment modality, DFI, survival, and presence of additional symptoms are recorded. DFI: disease-free interval; survival based on time from metastasis to death; SM: symptoms of metastasis.

est survival period from metastasis to death was 471 months in the same group with the DFI of 156 months. The two patients were treated with radiotherapy alone (Table 1).

\section{Discussion}

Although primary malignancies of the thymus are uncommon, the most common histological type is thymoma, a neoplasm of the thymic epithelial cells that is often associated with multiple autoimmune diseases [4]. Thymomas can be classified as "malignant" or "benign" based on evidence for capsular invasion. MTs typically spread to the lungs and the nearby mediastinal structures via direct growth or local extension [1].

In 2004, the World Health Organization (WHO) categorized thymic tumors as types A, AB, B1, B2, B3 and carcinoma [2]. Type A tumors consist of epithelial cells that are oval or spindle-shaped without atypia or lymphocytes, type $\mathrm{AB}$ tumors contain lymphocytes, and types B1-B3 tumors are made up of round epithelial cells with increasing proportions of atypia [3]. Unfortunately, this system does not appear to differentiate the patients who are at risk for metastatic spread as noted by Vladisla et al [2]

Rarely, thymomas can become malignant and metastasize. Based on SEER data, we have shown that the overall incidence of MT is increasing in the US, with a significant male predominance. Furthermore, we have documented 25 cases of metastatic thymoma to the abdomen and extrapolated the incidence of abdominal metastasis to be $0.92 \%$ of all MTs. We have also shown that $50 \%$ of patients with abdominal metastasis will present with symptoms and that pain is the most frequent complaint. Based on the data presented by Vladisla et al [2], the WHO classification does not predict abdominal spread. Previous literature has reported the most common routes of spread to be lymphatic and hematgenous [5]. Reviewing the available data, we have shown a wide variety of DFI for these patients, as well as the numerous different modalities of treatment.

Our findings have shown an increasing incidence of MT throughout the US. In 1973, there were 17 cases reported. In 2008, there were 90 cases reported to the SEER database. Furthermore, we have shown a significant male predominance ( 0.23 vs. 0.17 per 100,000 persons, 846 vs. $742, \mathrm{P}=0.0002$ ). Engels et al [1] provide a potential explanation for the higher incidence rate in males, suggesting an increased prevalence of occupational exposure. While other potential theories have been made, the epidemiological data comparing risk factors and genetic predispositions to thymoma are limited. Previous studies have found that thymoma incidence tends to be higher in Asians/Pacific Islanders, as well higher in blacks versus whites, suggesting the presence of genetic risk factors [6].

In the face of the rising overall incidence of MT, there has become a need for a formal guideline outlining routine screening and treatment protocols. According to our retrospective review, just under $50 \%$ (46\%) of patients with abdominal metastasis presented with symptoms. We recommend that patients with metastatic thymoma get routine imaging to look for extrathoracic metastasis irrespective of symptoms. As patients in our review had a wide variety of DFI, screening must be continued for quite some time to ensure recurrence does not occur.

Although progress has been made, little evidence exists that defines the best clinical practice in thymoma treatment [3]. Our review found a significant variation in the treatment strategies used over the course of time. While complete surgical resection remains the main goal in thymoma treatment, prospective randomized trials conducted in the past 10 - 15 years have demonstrated thymoma sensitivity to chemotherapy and radiation in cases where complete resection is not possible [7]. Radiation therapy has achieved high survival rates, but has been shown to be less efficacious if not proceeded by complete tumor resection. Therefore, pre-operative chemotherapy followed by post-operative radiotherapy could increase complete resection rate and potentially improve survival time in the treatment of the primary tumor [8].

Lucchi et al [7] suggest reoperation for thymoma recurrences to be feasible and relatively safe. Since other treatment 
modalities are not curative, we believe surgery should still be a mainstay in the treatment algorithm for metastatic disease. Our study demonstrates that the multimodality treatment (surgery, chemotherapy and radiotherapy) has a longer DFI than the surgery and chemotherapy alone groups.

Historically, DFI has been shown to have a strong correlation with survival, number of metastases, and tumor load [8]. In this study, DFI is a more appropriate way to objectively compare each treatment modality as survival time contains confounding variables such as age of diagnosis and comorbid conditions. We believe that the DFI is the best predictor of survival in patients with MT as it describes the tumor biology and aggressiveness.

Finally, this literature review lacks a great deal of detail into each patient's primary treatment, follow-up schedule, and available options. We suggest that a national treatment database be created to pool the available data and follow patients through the course of their disease.

\section{Conclusions}

Our study is one of the first to document the incidence of metastatic MT to the abdomen. Our retrospective analysis stresses the importance of the multimodal screening and treatment approaches.

\section{Conflict of Interest}

None.

\section{References}

1. Engels EA, Pfeiffer RM. Malignant thymoma in the United States: demographic patterns in incidence and associations with subsequent malignancies. Int $\mathrm{J}$ Cancer. 2003;105(4):546-551.

2. Vladislav T, Jain RK, Alvarez R, Mehta RJ, Gokmen-Polar Y, Kesler KA, Henley JD, et al. Extrathoracic metastases of thymic origin: a review of 35 cases. Mod Pathol. 2012;25(3):370-377.

3. Falkson CB, Bezjak A, Darling G, Gregg R, Malthaner R, Maziak DE, Yu E, et al. The management of thymoma: a systematic review and practice guideline. J Thorac Oncol. 2009;4(7):911-919.

4. Wang LS, Huang MH, Lin TS, Huang BS, Chien KY. Malignant thymoma. Cancer. 1992;70(2):443-450.

5. Bott-Kothari T, Aron BS, Bejarano P. Malignant thymoma with metastases to the gastrointestinal tract and ovary: a case report and literature review. Am J Clin Oncol. 2000;23(2):140-142.

6. Marom EM. Imaging thymoma. J Thorac Oncol. 2010;5(10 Suppl 4):S296-303.

7. Lucchi M, Ambrogi MC, Duranti L, Basolo F, Fontanini G, Angeletti CA, Mussi A. Advanced stage thymomas and thymic carcinomas: results of multimodality treatments. Ann Thorac Surg. 2005;79(6):1840-1844.

8. Multimodal management of stage III-IV malignant thymoma: role of neoadjuvant approaches. 2002. ASCO Annual Meeting. Presenter: Sergio Bretti. Session: Lung Cancer. 